\title{
¿ES LA CISTECTOMÍA RADICAL LAPAROSCÓPICA EL NUEVO STANDARD DE ORO PARA EL CÁNCER DE VEJIGA?
}

\author{
Octavio A. Castillol, 2,3, Gonzalo Vitagliano ${ }^{3}$ e Ivar Vidal-Mora'. \\ 'Unidad de Urología Clínica Indisa. Escuela de Medicina. Facultad de Ciencias de la Salud. Universidad Andrés \\ Bello. \\ ${ }^{2}$ Facultad de Medicina. Universidad de Chile. Santiago. Chile. \\ ${ }^{3}$ Servicio de Urologia. Hospital Alemán de Buenos Aires. Argentina.
}

\begin{abstract}
Resumen.- OBJETIVO: Presentar la serie de un centro Latinoamericano de laparoscopia urológica avanzada y comparar las ventajas contra la cirugía tradicional.

MÉTODOS: En un periodo de 7 años, se efectuaron un total de 85 cistectomías laparoscópicas con derivación urinaria efectuada por vía extracorpórea en el 92\% de los casos. Se realizaron 14 exanteraciones anteriores, 50 cistoprostatectomías radicales, 7 cistectomías radicales y 14 cistectomías simples. La relación hombre mujer fue de 3:1. La edad promedio fue de 63 años (rango 29 a 83). El índice de masa corporal (IMC) promedio, fue de $28 \mathrm{~kg} / \mathrm{m} 2$ (rango 20 a 47). Se analizan los resultados perioperatorios y a largo plazo obtenidos con esta técnica.
\end{abstract}

\section{CORRESPONDENCIA}

Octavio A. Castillo

Av. Apoquindo 3990, Of. 809

Las Condes.

Santiago de Chile.

Código postal: 7550112

octaviocastillo@vtr.net

Trabajo recibido: 27 de julio 2008.
RESULTADOS: Los 85 procedimientos se completaron por vía laparoscópica en forma completa. Se realizaron 42 neovejigas ortotópicas; 13 tipo Santiago, 16 tipo Studer, 12 tipo Fontana y 1 Le Bag. También se realizaron 24 conductos ileales, 10 Indianas y 9 Mainz II estos últimos enteramente por vía laparoscópica. El tiempo operatorio y sangrado promedio fueron de $279 \mathrm{mi}-$ nutos (rango 180 a 375) y $436 \mathrm{ml}$ (rango 50 a 1.500 ) respectivamente. Hubo 8 pacientes (17\%) con complicaciones intra o perioperatorias; 5 lesiones vasculares, 2 evisceraciones y 2 cuadros sépticos. Hubo 7 complicaciones tardías (9\%); 3 sepsis urinarias, 1 estenosis ureteral, 2 roturas espontáneas de reservorio y 1 isquemia mesentérica. El tiempo promedio de internación fue de 8,8 días (rango de 4 a 28). No hubo mortalidad operatoria. El seguimiento promedio fue de 18 meses con un rango de 2 a 68 meses. Diez pacientes (13\%) presentaron progresión y muerte por la enfermedad.

CONCLUSIÓN: La cistectomía radical laparoscópica presenta bajo sangrado operatorio, rápida recuperación del tránsito intestinal y pronta externación. No obstante ser un procedimiento reproducible, requiere de una larga curva de aprendizaje lo cual limita su difusión.

Palabras clave: Cáncer de vejiga. Cistectomía radical. Laparoscopía.

Summary.- OBJECTIVES: The experience of a renowned Latin American laparoscopic center is reported and the differences with the open technique are thoroughly discussed.

METHODS: During a 7 year period a total of 85 laparoscopic cystectomies were performed, in 92\% urinary 
diversion was performed extracorporeally. This accounted for: 14 anterior exenterations, 50 radical cystoprostatectomies, 7 radical cystectomies and 14 simple cystectomies. Male to female ratio was 3:1. Mean patient age was 63 years (range 29 to 83). Mean Body Mass Index (BMI) was $28 \mathrm{~kg} / \mathrm{m} 2$ (range 20 to 47). Operative data and long term results are analyzed.

RESULTS: All 85 procedures were completed laparoscopically without the need for conversion to open surgery. Orthotopic neobladder, Santiago pouch, Studer, Fontana and Le Bag were performed in 42, 13, 16, 12 and 1 case respectively. Ileal conduit, Indiana pouch and Mainz II were employed in 24, 10 and 9 cases respectively. All Mainz II were performed intracorporeally. Mean operative time and blood loss were 279 minutes (range 180 to 375 ) and $436 \mathrm{ml}$ (range 50 to 1.500 ) respectively. A total of 8 patients (1 1\%) presented perioperative complications: 5 vascular lesions, 2 eviscerations and 2 septicemias. Delayed complications were observed in 7 cases (9\%); 3 urinary sepsis, 1 ureteral stenosis, 2 spontaneous ruptures and 1 mesenteric ischemia. Mean hospital stay was 8.8 days (range de 4 to 28). There was no operative mortality. Mean follow-up was 18 months (range 2 to 68 months). Ten patients (13\%) presented disease progression and death.

CONCLUSIONS: Laparoscopic radical cystectomy is associated with diminished operative bleeding, time to oral intake and hospital stay. Though this is a reproducible technique it demands a very long learning curve.

Keywords: Bladder cancer. Radical cystectomy. Laparoscopy.

\section{INTRODUCCIÓN}

Son indiscutibles las ventajas que presenta el abordaje laparoscópico sobre la cirugía a cielo abierto. La restitución temprana de la dieta, el menor dolor postoperatorio, la deambulación precoz y la externación temprana han hecho que la laparoscopía sea utilizada para muchos procedimientos (1).

En urología, muchas técnicas han sido estandarizadas por vía laparoscópica facilitando su divulgación y aprendizaje, llegando incluso a convertirse en el "gold standard" como es el caso de la suprarrenalectomía laparoscópica para el tratamiento de la patología benigna de la glándula suprarrenal (2).

A esta evolución no ha escapado la cirugía oncológica urológica y procedimientos como la nefrectomía radical, la nefrectomía parcial, la linfadenectomía lumboaórtica y la prostatectomía radical se realizan en forma laparoscópica con los mismos resultados oncológicos que la cirugía abierta $(3,4)$.

La cistectomía radical sigue siendo el "standard of care" para el tratamiento del cáncer de vejiga músculo-invasor no metastático $(5,6)$. No obstante, recientemente se han publicado estudios que comienzan a presentar a la cistectomía radical laparoscópica como una opción viable y segura para el tratamiento de este grupo de pacientes (7). A pesar de presentar ventajas considerables, la complejidad de esta técnica exige del cirujano habilidades laparoscópicas avanzadas las cuales solo se obtienen luego de superar una larga curva de aprendizaje. Consiguientemente la escasa reproducibilidad de esta técnica ha sido utilizada como argumento para poner en duda la existencia de verdaderas ventajas por sobre el abordaje convencional.

\section{MATERIAL Y MÉTODOS}

En un periodo de 7 años comprendido entre enero de 2000 y julio de 2007, se efectuaron un total de 85 cistectomías laparoscópicas con derivación urinaria efectuada por vía extracorpórea en el $92 \%$ de los casos. De estas, 14 fueron exanteraciones anteriores, 50 cistoprostatectomías radicales, 7 cistectomías radicales y 14 cistectomías simples. A todos los pacientes intervenidos por motivos oncológicos se les efectuó una linfadenectomía pelviana completa en forma laparoscópica. La relación hombre mujer fue de 3:1 (57 hombres / 19 mujeres). La edad promedio fue de 63 años (rango 29 a 83 años). El riesgo quirúrgico anestésico promedio según la clasificación de la Sociedad de Anestesiología Americana (ASA) fue ASA 2. El índice de masa corporal (IMC) promedio, fue de $28 \mathrm{~kg} / \mathrm{m}^{2}$ (rango 20 a $47 \mathrm{~kg} / \mathrm{m}^{2}$ ). De estos pacientes, 5 fueron sometidos a radioterapia en forma previa a la cirugía. A su vez las comorbilidades mas frecuentes en orden decreciente fueron; hipertensión arterial (28 casos), diabetes mellitas ( 10 casos), enfermedad pulmonar obstructiva crónica (9 casos), coronariopatía crónica (2 casos) e insuficiencia renal crónica (1 caso). Los datos demográficos de los pacientes son detallados en la Tabla I.

A todos los pacientes sometidos a cistectomía radical por cáncer vesical se les efectuó una resección endoscópica vesical previa para una adecuada estadificación local. Todos los pacientes fueron sometidos a un examen clínico completo, incluyendo un estudio endoscópico de la vejiga y uretra, tomografía computada de abdomen, radiografía de tórax, centellograma óseo y un estudio hematológico completo. 
TABLA I. DATOS DEMOGRÁFICOS.

\begin{tabular}{|l|c|}
\hline Datos demográficos & $N=85$ \\
\hline Edad & 63 años (29 a 83) \\
\hline Relación hombre/mujer & $3: 1$ \\
\hline IMC & $28 \mathrm{Kg} / \mathrm{m}(20$ a 47$)$ \\
\hline ASA & 2 (1 a 3) \\
\hline Cirugías realizadas & 85 \\
Cistectomía & 14 \\
Cistectomía radical & 7 \\
Cistoprostatectomía radical & 50 \\
Exanteración anterior & 14 \\
\hline
\end{tabular}

\section{Técnica quirúrgica:}

Se realiza preparación intestinal el día anterior a la cirugía con 2 frascos de fosfo-don $\circledast$. Seis horas antes se administra una dosis de heparina de bajo peso molecular y en la inducción anestésica se administra una cefalosporina de primera generación y metronidazol.

El paciente se coloca en una posición de litotomía modificada con los brazos adheridos al cuerpo. Se coloca apoyo en los hombros para permitir una posición de Trendelemburg máxima (Figura. 1). El cirujano se coloca del lado izquierdo del paciente. Se realiza el neumoperitoneo con punción con aguja de Veress a través de una incisión supraumbilical, hasta una presión de $15 \mathrm{mmHg}$. Se coloca un primer trocar supraumbilical de $10 \mathrm{~mm}$ para la óptica, y 4 trócares adicionales de trabajo: 2 de $10 \mathrm{~mm}$ pararectales y 2 de $5 \mathrm{~mm}$ para umbilicales (Figura 2).

La cirugía comienza realizando una sección del peritoneo del fondo de saco recto-vesical. La incisión peritoneal se continúa en forma bilateral siguiendo una línea imaginaria entre los vasos espermáticos y el ligamento umbilical bilateral formando una " $\mathrm{H}$ ". Se seccionan los uréteres entre clips, enviando un segmento para biopsia por congelación. El clipaje del uréter evita la contaminación del campo quirúrgico con orina y permite una dilatación que facilita el neoimplante posterior. La linfadenectomía se realiza comenzando desde encima de la bifurcación ilíaca hasta el extremo distal de los vasos iliacos externos, incluyendo el paquete obturatríz y siendo los límites laterales el nervio genito-femoral y la vejiga.

Se secciona la hoja posterior de la fascia de Denovilliers, separando la vejiga del recto y se procede a la disección e identificación de los pedículos vesicales, los cuales se pueden seccionar con Endogia $^{T M}$ (Ethicon, Endosurgery), Ligasure ${ }^{T M}$ (Valley Lab), Bisturí Armónico (Ethicon Endosurgery) o coagulación bipolar (Gyrus ${ }^{\top \mathrm{M}}$ ).

Para la disección del ápex se realiza una apertura de la fascia endopélvica de ambos lados y se pasa un punto de $V y c r i l \circledast 0$ para control del

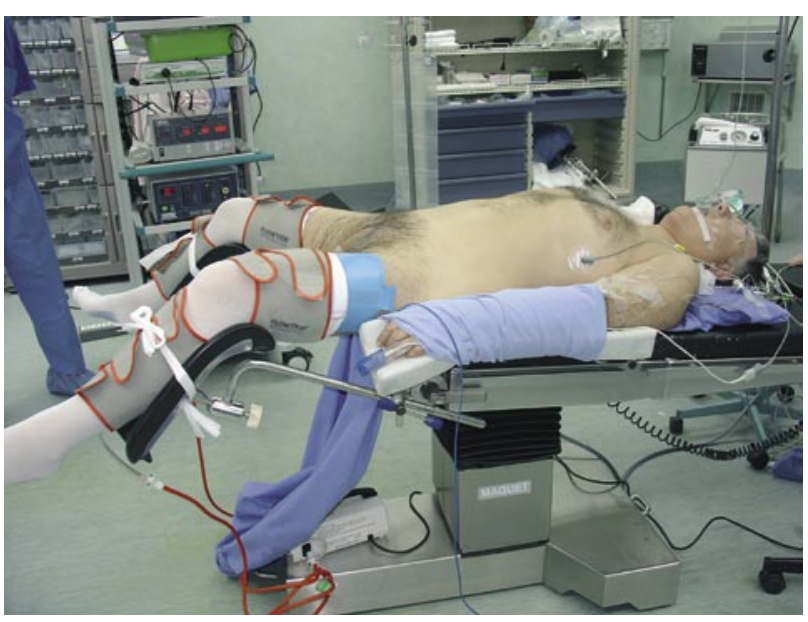

FIGURA 1. Posición del paciente. 
complejo venoso dorsal. Se completa la disección en forma retrógrada y la pieza es colocada en una bolsa y se extrae por una incisión media longitudinal de $5-6 \mathrm{~cm}$ (Figura 3).

El segmento intestinal, previamente seleccionado por laparoscopía, se extrae a través de la incisión, efectuando la anastomosis intestinal, la derivación urinaria elegida y los neoimplantes ureterales en forma totalmente extracorpórea. En el caso de una neovejiga ortotópica, se cierra la incisión media y se realiza la anastomosis neovejiga-uretra con 6 puntos intracorpóreos de $\mathrm{Vycril}$ @ 2-0. Al término de la cirugía se deja un drenaje aspirativo por contrabertura izquierda y los tutores ureterales por contrabertura derecha, excepto en los conductos ileales donde se sacan a través del ostoma.

\section{RESULTADOS}

Los 85 procedimientos se completaron por vía laparoscópica en forma completa, no necesitando conversión a cirugía abierta. Se realizaron 14 exanteraciones anteriores, 50 cistoprostatectomías radicales, 7 cistectomías radicales y 14 cistectomías simples. El tipo de derivación realizada fue una neovejiga ortotópica en 42 casos de los cuales 13 fueron tipo Santiago, 16 tipo Studer, 12 tipo Fontana y 1 Le Bag. También se realizaron 24 conductos ileales, 10 Indianas y 9 Mainz II estos últimos enteramente por vía laparoscópica. El tiempo operatorio total varió entre 180 y 375 minutos, con un promedio de 279 minutos. En la Tabla II se detallan los tiempos

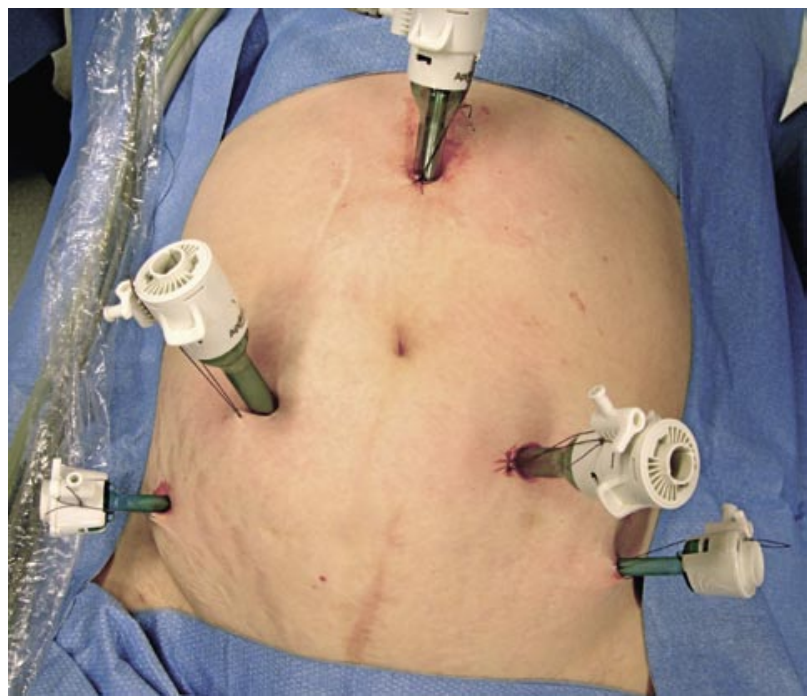

FIGURA 2. Posición de los puertos de trabajo. operatorios parciales. El sangrado intraoperatorio osciló entre 50 y $1.500 \mathrm{ml}$, con un promedio de $436 \mathrm{ml}$ y un índice de transfusión de 13\%.

Un total de 8 pacientes (11\%) presentaron complicaciones intra o perioperatorias. Estas fueron 5 lesiones vasculares resueltas en forma intracorpórea (arteria ilíaca 1, pedículo vesical 2, arteria epigástrica 1 y vena ilíaca común 1), 2 evisceraciones que requirieron reoperación y 2 cuadros de sepsis tratados médicamente. Ocurrieron 7 complicaciones tardías $(9 \%)$. Estas fueron 3 sepsis urinarias controladas médicamente, 1 estenosis ureteral resuelta mediante dilatación neumática, 2 roturas espontáneas de reservorio a los 15 y 48 meses respectivamente y 1 isquemia mesentérica en el contexto de un Linfoma no Hodgkin que desencadenó la muerte del paciente.

En todos los pacientes se inició la deambulación dentro de 12 horas de la cirugía y fueron realimentados a las 36 horas. Ningún paciente requirió opiáceos para el manejo del dolor, y sólo se utilizó una infusión continua de Ketorolaco las primeras 24 horas. El tiempo promedio de internación fue de 8,8

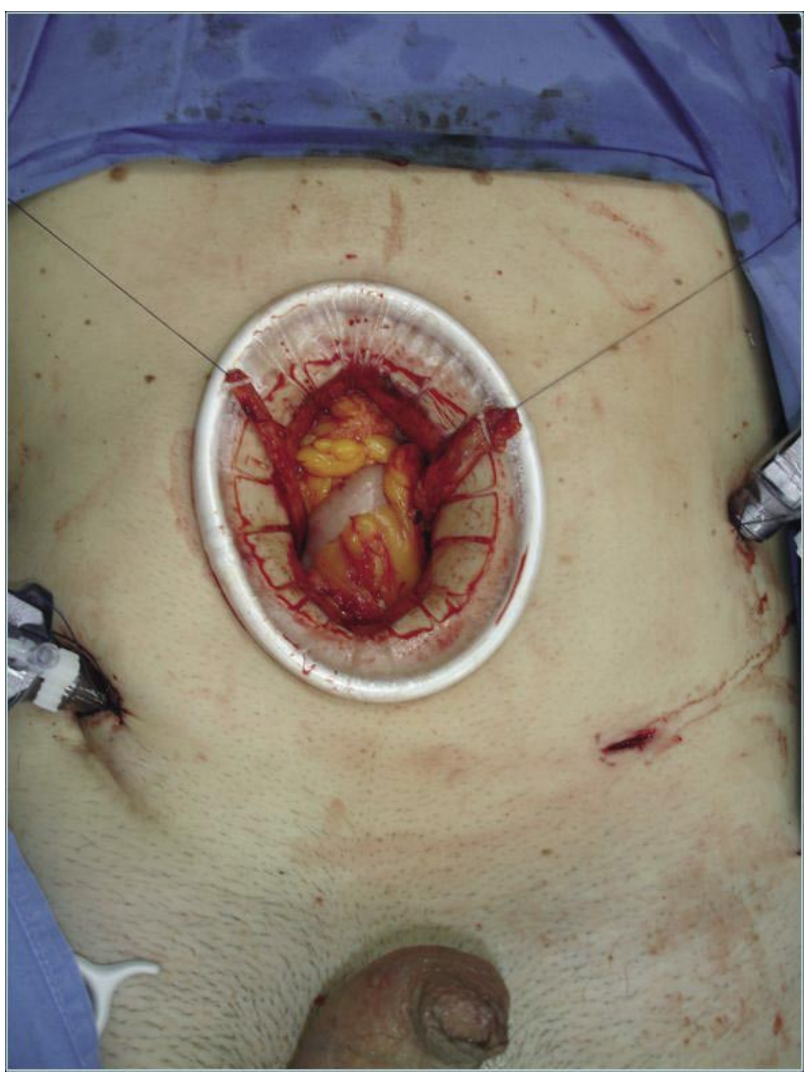

FIGURA 3. Laparotomía infraumbilical para extracción de espécimen y derivación extracorpórea. 
días, con un rango de 4 a 28 días. No hubo mortalidad operatoria. El estadío tumoral determinado por anatomía patológica fue $\mathrm{Tx}, \mathrm{TO}, \mathrm{Tis}, \mathrm{T1}, \mathrm{T} 2 \mathrm{a}$, T2b, T3a, T3b y T4a en 1, 3, 11, 6, 10, 9, 18, 4 y 3 casos respectivamente. El número promedio de adenopatías resecadas fue de 17 ganglios con un rango entre 10 y 28 ganglios. Tres pacientes presentaron metástasis ganglionares, dos pacientes T3a y un paciente T3b. El seguimiento promedio fue de 18 meses con un rango de 2 a 68 meses. Durante este tiempo 10 pacientes $(13 \%)$ presentaron progresión y muerte por la enfermedad. De estos, 4 pacientes presentaron recidiva local de los cuales 2 presentaron metástasis a distancia. Un total de 6 pacientes evolucionó con únicamente con metástasis a distancia. El tiempo promedio de supervivencia para los pacientes con recidiva local, recidiva local más metástasis a distancia y metástasis a distancia únicamente fue de 17, 11 y 11 meses respectivamente. La Tabla III resume los resultados perioperatorios.

\section{DISCUSIÓN}

Fueron Parra et al. (8) en 1992 quienes publicaron la primera cistectomía laparoscópica por un piocistos en una vejiga retenida. Tres años mas tarde Sánchez de Badajoz y col reportaron la primera cistectomía radical laparoscópica por cáncer de vejiga músculo invasor (9). A estos autores les siguieron otros que lentamente fueron perfeccionando las técnicas hasta llegar a la realización completa de la cirugía en forma intracorpórea (10).

Actualmente diversos centros alrededor del mundo, especializados en laparoscopia urológica avanzada, presentan series que promedian los 30 casos $(7,11,12)$. A su vez, existen grupos de investigación multicéntricos que se encuentran evaluando los resultados de este procedimiento a corto y largo plazo para poder determinar si existen concretas ventajas de realizar esta cirugía por vía laparoscópica (13).

Pese a que existen casos aislados y pequeñas series reportadas en la literatura internacional, el procedimiento enteramente laparoscópico es infrecuente ya que demanda de un tiempo operatorio prolongado y son limitadas las opciones reconstructivas que pueden ser efectuadas totalmente en forma intracorpórea (10). Es por esto que en forma genérica, se entiende por cistectomía radical laparoscópica la técnica que involucra la creación del reservorio o neovejiga en forma extracorpórea mientras que el resto del procedimiento se efectúa laparoscópicamente. Se debe entender que tanto la cistectomía convencional como la laparoscópica requieren de laparotomía y consiguiente exposición de las asas intestinales para realizar el reservorio y la reconstitución del tránsito.

Los primeros en comparar ambos procedimientos fueron Basillote et al. (14) los autores cotejaron los resultados de 19 pacientes en forma laparoscópica con 13 operados en forma clásica. No existió diferencia significativa en el tiempo operatorio, sangrado, ni en el índice de complicaciones. No obstante, el uso de analgésicos, el tiempo necesario para comenzar la ingesta oral y los días de hospitalización fueron significativamente menores para el grupo laparoscópico. Posteriormente, Taylor et al. (15) en 2004 publicaron un total de 36 cistectomías, en las últimas 16 la derivación urinaria fue mediante un conducto ileal. De estos 16 procedimientos, 8 fueron realizados en forma laparoscópica utilizando asistencia manual. Al comparar ambos grupos, los autores

TABLA II. TIEMPOS OPERATORIOS PARCIALES.

\begin{tabular}{|l|c|}
\hline \multicolumn{1}{|c|}{ Procedimiento } & Tiempo operatorio promedio (minutos) \\
\hline Linfadenectomía bilateral & $58(35-85)$ \\
\hline Cistectomía & $63(40-120)$ \\
\hline Derivación urinaria* & $76(35-150)$ \\
\hline Anastomosis uretral & $38(20-70)$ \\
\hline Tiempo total & $278(180-420)$ \\
\hline
\end{tabular}

* NOTA: Dentro de el tiempo de derivación urinaria se incluyen los 6 Mainz II. 
encontraron diferencias estadísticamente significativas a favor del grupo laparoscópico. La disminución en el sangrado intraoperatorio, requerimiento de analgesia, días de hospitalización y la pronta realimentación favoreció al grupo laparoscópico. No obstante, el corto seguimiento previno a los autores de comparar los resultados oncológicos a largo plazo, este estudio demostró la existencia de una importante ventaja en términos de convalecencia postoperatoria para la cistectomía radical laparoscópica.

Pese a que en nuestro centro no se ha efectuado una comparación prospectiva y randomizada entre ambos procedimientos (cielo abierto y video asistido), creemos lícito contrastar nuestros resultados con los publicados en las principales series de cistectomía radical a cielo abierto.
Basillote et al. compararon ambos procedimientos en función del tiempo y no obtuvieron diferencias estadísticamente significativas luego de superada la curva de aprendizaje (14). En una reciente publicación, Gerullis et al reportan un tiempo promedio de 244 minutos en su serie de 34 pacientes (11). En nuestro caso particular se debe tener en cuenta que la cistectomía radical laparoscópica ha sido recientemente desarrollada y se encuentra en constante transformación, observándose significativas diferencias dentro de la misma serie. Esto hace que una comparación directa de un promedio de tiempos no sea totalmente representativa. Pese a esto en nuestra serie obtuvimos un tiempo operatorio promedio de $279 \mathrm{mi}-$ nutos (rango 180 a 375 minutos), lo cual se compara favorablemente con otras series laparoscópicas hasta ahora publicadas. Cabe destacar que dentro de estos

TABLA III. RESULTADOS PERIOPERATORIOS.

\begin{tabular}{|c|c|}
\hline Resultados perioperatorios & $N=85$ \\
\hline Sangrado & $436 \mathrm{ml}(50$ a 1500$)$ \\
\hline Índice de transfusión & $13 \%$ \\
\hline Complicaciones & $20 \%$ \\
\hline Perioperatorias & $11 \%$ \\
\hline Lesiones vasculares & 5 \\
\hline Evisceraciones & 2 \\
\hline Sepsis & 2 \\
\hline Tardías & $9 \%$ \\
\hline Sepsis & 3 \\
\hline Rotura espontánea de reservorio & 2 \\
\hline Isquemia mesentérica & 1 \\
\hline Estadía hospitalaria & 8,8 días (4 a 28) \\
\hline Tiempo de seguimiento & 18 meses (2 a 68) \\
\hline \multicolumn{2}{|l|}{ Sobrevida } \\
\hline Con recidiva local & 17 meses \\
\hline Con recidiva local y a distancia & 11 meses \\
\hline Con recidiva a distancia unicamente & 11 meses \\
\hline
\end{tabular}


tiempos están también las 6 derivaciones efectuadas enteramente por vía laparoscópica.

Con respecto al sangrado intraoperatorio y el consiguiente índice de transfusión, existe una clara ventaja del abordaje laparoscópico sobre el abierto. Haber y Gill en una serie de 37 pacientes reportaron un promedio de $608 \mathrm{ml}$ (rango 150 a $2000 \mathrm{ml}$ ) con un índice de transfusión del 13\% (7). Gerullis y col reportan un sangrado promedio de $325 \mathrm{ml}$ (rango 100 a $700 \mathrm{ml}$ ) con un índice de transfusión de $5.9 \%$ para su serie e 34 pacientes (11). En la serie actual observamos un sangrado promedio de $436 \mathrm{ml}$ (rango 50 a $1500 \mathrm{ml}$ ) con un $13 \%$ de índice de transfusión. La mayoría de los autores encuentran un reducción de aproximadamente $400 \mathrm{ml}$ con la técnica laparoscópica (16). Esto se explicaría por la mejor visualización de los pedículos vasculares que permitiría una hemostasia más eficaz. Por otro lado, la presión positiva del pneumoperitoneo actuaría disminuyendo el sangrado intraoperatorio.

La vía laparoscópica al igual que la abierta pretende respetar los principios básicos de la cirugía oncológica. Es por ello que los pasos quirúrgicos empleados en ambas cirugías son similares. De esto se desprende que las posibles complicaciones de la vía laparoscópicas estarán únicamente relacionadas con el tipo de abordaje y no con una cirugía diferente. Las complicaciones reportadas por Ukimura et al. (17) sobre la experiencia inicial de 21 casos en la Cleveland Clinic muestran un $29 \%$ de complicaciones mayores (requirieron reoperación) y un $45 \%$ de complicaciones menores (relacionadas a íleo prolongado). En la serie actual encontramos un $11 \%$ de complicaciones intra o perioperatorias y un $9 \%$ de complicaciones tardías.

El íleo asociado a la cistectomía radical es producto de la exteriorización y desfuncionalización intestinal y la utilización de opiáceos durante el postoperatorio. En muchos casos es necesaria la institución de nutrición parenteral para prevenir la desnutrición del paciente. Es importante recalcar que algunos pacientes son sometidos a la cistectomía luego de haber recibido tratamientos quimioterapéuticos neoadyuvantes y por consiguiente se encuentran en un estado hipoproteinémico en forma previa a la cirugía. A su vez no es despreciable la importante pérdida de linfa, con el consecuente detrimento nutricional, luego de una linfadenectomía iliobturatríz bilateral extensa. Lo antes mencionado asociado a un íleo prolongado puede comprometer seriamente la evolución postoperatoria de esta cirugía.

Ambas técnicas presentan un tiempo de exteriorización de las asas intestinales. No obstante la di- ferencia significativa en el tamaño de la laparotomía se asocia a una menor exposición del contenido de la cavidad abdominal y a su vez esta directamente relacionada con una menor utilización de derivados opiáceos. Traduciéndose esto en un menor íleo postoperatorio y una temprana reanudación de la ingesta oral. Basillote et al. demostraron una diferencia significativa en el tiempo necesario para realimentar al paciente $(2,8$ días vs 5 días, $\mathrm{P}<0.004)(14)$.

Existe una relación directa entre el tamaño y ubicación de una incisión quirúrgica con el dolor postoperatorio. En el caso de la cistectomía radical, el empleo de la laparoscopía posibilita la realización de una incisión que promedia los $8 \mathrm{~cm}$. Esta es un $60 \%$ más pequeña que la utilizada para realizar la cirugía en forma convencional. Por otro lado, al no ser sometida la pared intestinal a una prolongada separación, la incidencia de dolor postoperatorio es menor. Por consiguiente, la deambulación es precoz y la necesidad de analgésicos opiáceos se ve disminuida.

La estadía hospitalaria estará directamente relacionada con la incidencia de complicaciones, la precocidad de la ingesta y el dolor postoperatorio. Los pacientes que presenten menor íleo postoperatorio y deambulación precoz serán pasibles un alta temprana. No obstante no se debe minimizar las complicaciones inherentes a todo reservorio intestinal como ser la obstrucción del mismo por moco intestinal y los desequilibrios hidroelectrolíticos. Estos últimos pueden retrasar la externación del paciente independientemente de la técnica empleada. El tiempo promedio de internación en nuestra serie fue de 8,8 días, no obstante, el rango osciló entre 4 y 28 días.

No es despreciable la diferencia en costos que presentan ambos procedimientos. El costo del instrumental necesario para realizar la vía laparoscópica es alto. No obstante, los insumos laparoscópicos han disminuido su precio y pueden ser reutilizados en más de una oportunidad. Por otro lado pese a presentar un mayor costo quirúrgico, la menor convalecencia postoperatoria de la laparoscopía se asocia a una disminución en los costos de internación.

La incisión mediana utilizada para la extracción de la pieza quirúrgica y exteriorización de las asas intestinales admite la cómoda confección de todos los reservorios hasta ahora conocidos. Esto se explica por el hecho de que los uréteres sean liberados ampliamente en sentido cefálico asociado a la posibilidad de movilizar por vía laparoscópica la porción de intestino seleccionada con tal fin. Tampoco existe limitación para la realización de neo-vejigas ortotópicas. 
Abreu et al. (18) reportaron la realización extracorpórea de un reservorio en $\mathrm{V}$ tipo Fontana. Los autores presentaron su experiencia inicial con tres pacientes con excelentes resultados. En nuestra serie esta técnica ha sido empleada en 12 ocasiones. Cabe mencionar que pese a la utilización de sutura mecánica irreabsorvible, no presentamos casos de litiasis asociadas a las mismas. Esto podría ser explicado por la epitelización que se produce sobre la línea de sutura, quedando esta aislada de la orina.

Con respecto a la seguridad oncológica, recientemente Haber y Gill (7) presentaron el primer reporte que existe en la literatura urológica de seguimiento mayor a 5 años luego de cistectomía radical laparoscópica. En su trabajo los autores reportaron 37 pacientes sometidos a cistectomía radical laparoscópica con derivación urinaria en un periodo de 6 años. Al $70 \%$ de los pacientes (26 casos) se le efectuó una linfadenectomía pelviana ampliada. El 86\% fueron carcinomas transicionales de alta grado (GIII en $78 \%$ ) y de estadio superior a pT2 (70\%). El tiempo promedio de seguimiento fue de 31 meses (rango 1 a 66 meses). La sobrevida global, la sobrevida cáncer específica y la sobrevida libre de recurrencia fue de $63 \%, 92 \%$ y $92 \%$ respectivamente. Los autores concluyeron que la cistectomía radical laparoscópica brinda resultados oncológicos comparables con las series contemporáneas de cistectomía abierta.

\section{CONCLUSIONES}

La cistectomía radical laparoscópica presenta bajo sangrado operatorio, rápida recuperación del tránsito intestinal y un alta hospitalaria precoz. También exhibe resultados a corto y largo plazo comparables con la cirugía abierta. No obstante para ser un procedimiento reproducible, requiere de una larga curva de aprendizaje lo cual limita su difusión.

\section{BIBLIOGRAFÍA y LECTURAS RECOMENDADAS ( ${ }^{*}$ lectura de interés $y^{* *}$ lectura fundamental)}

*1. Jonson B, Zethraeus N. Costs and benefits of laparoscopic surgery: a review of the literature. Eur. J. Surg. 2000; 585:48-56.

2. Guazzoni G, Cestari A, Montorsi F, Bellinzoni P, Centemero A, Naspro R, et al. Laparoscopic treatment of adrenal diseases: 10 years on. BJU International, 2004; 93:221-7.

**3. Rassweiler J, Tsivian A, Kumar AV, Lymberakis C, Schulze M, Seeman O, et al. Oncological safe- ty of laparoscopic surgery for urological malignancy: experience with more than 1000 operations. J. Urol. 2003; 169:2072-5.

4. Tooher R, Swindle P, Woo H, Miller J, Maddern G. Laparoscopic Radical Prostatectomy for Localized Prostate Cancer: a systematic review of comparative studies. J. Urol. 2006; 175:2011-7.

*5. Ghoneim MA, El-Mekresh MM, El-Baz MA. Radical cystectomy for carcinoma of the bladder: critical evaluation of the results in 1026 cases. J. Urol. 1997; 158:393-9.

6. Stein JP, Lieskovsky G, Cote R. Radical cystectomy in the treatment of invasive bladder cancer: long-term results in 1054 patients. J. Clin. Oncol. 2001; 19:666-75.

**7. Haber GP, Gill IS. Laparoscopic radical cystectomy for cancer: oncological outcomes at up to 5 years. BJU Int. 2007; 100:137-42.

8. Parra RO, Andrés CH, Jones JP. Laparoscopic cystectomy: inicial report on a new treatment for the retained bladder. J Urol. 1992; 148:1140-3.

9. Sanchez de Badajoz E, Gallego Perales JL, Reche Rosado A. Laparoscopic cystectomy and ileal conduit: case report. J Endourol. 1995; 9:59.

**10. Puppo P, Naselli A. Laparoscopic radical cystectomy. Curr. Urol. Rep. 2005; 6(2):106-18.

11. Gerullis H, Kuemmel C, Popken G. Laparoscopic cystectomy with extracorporeal-assited urinary diversión: experience with 34 patients. European Urology, 2006; 51:193-8.

**12. Hrouda D, Adeyoju AB, Gill I. Laparoscopic radical cystectomy and urinary diversion: fad or future? BJU Int. 2004; 94:501-5.

**13. Haber G, Gill IS, Rozet F, Vallancien G, Piechaud $\mathrm{T}$, Gaston R, et al. International registry of laparoscopic radical cystectomy: report on 492 patients. J. Endourology, 2006; 1:8-23.

*14. Basillote JB, Abdelshehid C, Ahlering T, Shanberg A.Laparoscopic assisted radical cystectomy with ileal neobladder: a comparison with the open approach. J.Urol.2004; 172:489-93.

15. Taylor GD, Duchene DA, Koeneman KS. Handassisted laparoscopic cystectomy with minilaparotomy ileal conduit: series report and comparison with open cystectomy. J. Urol. 2004; 172: 1291-6.

16. Fergany AF, Novick AC, Gill IS. Laparoscopic urinary diversión. World J. Urol. 2000; 18:345-8.

17. Ukimura O, Moinzadeh A, Gill IS, Laparoscopic radical cystectomy and urinary diversión. Curr. Urol. Rep. 2005; 6:118-21.

18. Abreu SC, Araujo MB, Silveira RA. Laparoscopic-assisted radical cystectomy with u-shaped orthotopic ileal neobladder constructed using nonabsorbable titanium staples. J. Urol. 2006; 68(1):193-7. 